\title{
Evolving Treatment Paradigm in Metastatic Renal Cell Carcinoma
}

\author{
David M. Gill, MD, Neeraj Agarwal, MD, Ulka Vaishampayan, MD \\ Huntsman Cancer Institute, University of Utah, Salt Lake City, UT; Department of Internal \\ Medicine, University of Utah School of Medicine, Salt Lake City, UT; Karmanos Cancer Institute, \\ Wayne State University, Detroit, MI.
}

\begin{abstract}
The treatment paradigm for advanced and metastatic renal cell carcinoma (mRCC) has evolved rapidly since the arrival of targeted therapies and novel immunotherapies. mRCC was previously treated only with cytokines. However, discoveries of mutations affecting the von Hippel-Lindau tumor suppressor gene (leading to increased expression of VEGF and hypoxia inducible factor/ HIF-1) and of deregulations in the phosphatidylinositol-3 kinase/AKT/mTOR pathway (resulting in tumor angiogenesis, cell proliferation, and tumor growth) have led to the development of numerous targeted therapies. The U.S. Food and Drug Administration (FDA) has thus approved a total of nine targeted therapies since 2005, including VEGF tyrosine kinase inhibitors (sunitinib, pazopanib, axitinib, sorafenib, and lenvatinib), a monoclonal antibody targeting VEGF (bevacizumab), mTOR inhibitors (temsirolimus and everolimus), and a multityrosine kinase inhibitor (cabozantinib). Furthermore, the development of immune checkpoint inhibitors has again shifted the mRCC therapeutic landscape with the FDA's approval of nivolumab. Herein, we discuss the unprecedented changes in the field of clear cell histology mRCC in both the first-line and salvage settings, and we also discuss future therapies and recommend a treatment paradigm on sequencing of these agents.
\end{abstract}

Prior to 2005 when the FDA approved the first antiangiogenic agent in RCC, treatment of mRCC consisted solely of cytokines. The FDA approved high-dose interleukin-2 (HDIL-2) in 1992 for first-line treatment of mRCC, after preliminary data showed an overall response rate (ORR) of $15 \%$ as well as a $5 \%$ complete response (CR). ${ }^{1} \mathrm{~A}$ follow-up study reported a $7 \% \mathrm{CR}$, with a median duration of response of at least 80 months. ${ }^{2}$ However, given acute dose-limiting toxicities, inclusion criteria require excellent performance status and adequate organ function. ${ }^{1}$ In an attempt to decrease toxicity, Yang et al ${ }^{3}$ compared high- and low-dose interleukin-2 but, unfortunately, ORR was greater in the high-dose $\operatorname{arm}(21 \%$ vs. $13 \%, \mathrm{p}=$. 048). In addition, analysis from a 2017 prospective cohort (352 patients) 4 and a 2016 retrospective cohort (391 patients) ${ }^{5}$ suggests that the survival benefit with HDIL-2 may extend to many more patients. In addition to those with partial response and CR, stable disease (SD) as the best response to therapy was present for $39 \%$ and $32 \%$ of patients, respectively. Although SD is not historically categorized in HDIL-2 trials, the presence of SD was associated with a survival benefit in these two independent cohorts. ${ }^{4,5}$ Interferon-a

Corresponding author: Ulka Vaishampayan, MD, Karmanos Cancer Institute, Wayne State University, 4100 John R St., Detroit, MI 48201; vaishamu@karmanos.org.

Disclosures of potential conflicts of interest provided by the authors are available with the online article at asco.org/edbook. 
(hereafter referred to as interferon) had more modest outcomes (overall survival [OS] 2.5 months greater than placebo) without the durable responses of HDIL-2; however, interferon was better tolerated with broader eligibility criteria and therefore was the mainstay of treatment of most patients with mRCC prior to the advent of antiangiogenic therapy. ${ }^{6}$

\section{FIRST-LINE THERAPY SETTING}

\section{VEGF-Targeted Therapies}

VEGF-targeted therapies with tyrosine kinase inhibitors (TKIs) were developed as a result of improved understanding of von Hippel-Lindau gene mutations leading to the induction of angiogenic protein. ${ }^{7}$ VEGF-TKIs currently approved for mRCC include sunitinib, sorafenib, pazopanib, axitinib, cabozantinib, and lenvatinib (Fig. 1). Similarly, bevacizumab is a monoclonal antibody directed against the VEGF receptor (VEGFR). For over a decade, cytokines were the only approved treatment for mRCC. In 2005, sorafenib changed this paradigm, with the TARGET study showing improvement in progression-free survival (PFS) versus placebo in the second-line setting after cytokine therapy (5.5 vs. 2.8 months; $\mathrm{p}<.01$ ). ${ }^{8}$ Shortly thereafter, a landmark 2007 study by Motzer et $\mathrm{al}^{9}$ showed improved PFS with sunitinib compared with interferon in the first-line setting (11.0 vs. 5.0 months; $p<.001)$. In 2007, AVOREN trial investigators published a comparison of bevacizumab and interferon in combination versus interferon monotherapy. Again, the results nearly doubled the PFS of the comparator arm (10.2 vs. 5.4 months, $\mathrm{p}=.0001) .{ }^{10}$ In a phase III study published in 2010 (VEG105192), pazopanib was used for previously untreated patients and those who had progressed after cytokines. Compared with placebo, there was a 5-month improvement in PFS (9.2 vs. 4.2 months; $p$ <.0001). ${ }^{11}$ In 2009, the FDA approved both pazopanib and bevacizumab in combination with interferon (Table 1).

Since that time, no other agent has been approved for the first-line therapy setting. However, a recently published study ${ }^{24}$ may change this. Cabozantinib is a small molecule inhibitor targeting multiple tyrosine kinases, including VEGFR-2, hepatocyte growth factor (MET), and AXL. The Alliance A031203 CABOSUN trial compared cabozantinib to sunitinib for patients with previously untreated $\mathrm{mRCC}$ with poor and intermediate prognosis per International Metastatic Renal Cell Carcinoma Database Consortium (IMDC) criteria. Results showed improvement in PFS (8.2 vs. 5.6 months) and ORR (46\% vs. 18\%) and a $34 \%$ reduction in the rate of progression or death (adjusted hazard ratio [HR], $0.66 ; 95 \% \mathrm{CI}$, $0.46-0.95$; one-sided $\mathrm{p}=.012$ ) in the cabozantinib arm, with a similar incidence of grade 3 or 4 adverse events (AEs; $67 \%$ vs. $68 \%$ ). ${ }^{24}$ Preliminary data on OS, a secondary endpoint, revealed a $20 \%$ decrease in the rate of death with cabozantinib, although the study was not designed to show a difference in OS. An independent review for the primary endpoint of PFS is ongoing and if this confirms the findings then cabozantinib will be submitted to the FDA for consideration of approval as front-line therapy.

\section{mTOR Inhibitors in Front-Line Therapy}

Other discoveries showed that mutations in phosphatidylinositol-3 kinase, a kinase upstream of mTOR, were both common in mRCC and amenable to targeted therapy. ${ }^{25}$ In 2007, the FDA approved an mTOR inhibitor (temsirolimus) for patients with previously untreated 
mRCC who were in the poor prognosis category, based on a study showing improvement in OS compared with interferon (10.9 vs. 7.3 months; $\mathrm{p}=.008)$ in the Global ARCC trial.

Notably, although it was not the primary endpoint, there was only a modest improvement in PFS over interferon by independent radiographic assessment (5.5 vs. 3.1 months). The combination of interferon with temsirolimus was also evaluated but did not improve PFS or OS. ${ }^{12}$ With low response rates and modest clinical benefit, temsirolimus is not a widely used therapy in front-line mRCC management.

\section{Notable First-Line Studies Not Resulting in Changes in Regulatory Approval}

In a randomized study of sorafenib compared with interferon for previously untreated patients, there was no improvement in PFS (5.7 vs. 5.6 months). ${ }^{26}$ Another study investigated axitinib versus sorafenib for 288 previously untreated patients. There was an improvement in ORR (32\% vs. $15 \%$ ) and a nonsignificant trend toward improved PFS (median 10.0 vs. 6.5 months) favoring the axitinib arm. ${ }^{27}$ The TIVO-1 trial investigated VEGF-TKI tivozanib with sorafenib for 517 previously untreated patients. Although there was an improvement in PFS in the tivozanib arm (11.9 vs. 9.1 months; $p=.042)$, there was a trend toward longer OS in the sorafenib arm (29.3 vs. 28.8 months; $p=.105)$. However, this may have been complicated by notable cross-over differences in the two arms. Of 189 patients who discontinued tivozanib, only $36 \%$ received a second therapy and only $10 \%$ received another VEGF-directed therapy. Of 226 patients who discontinued sorafenib, 74\% received a second-line treatment and nearly $70 \%$ received tivozanib. ${ }^{28}$ Everolimus, an mTOR inhibitor, was compared with sunitinib for 471 previously untreated patients in the RECORD-3 study using a cross-over treatment design following disease progression. The primary endpoint was noninferiority of everolimus compared with sunitinib in the first-line therapy setting. In addition to inferior PFS in the everolimus arm (7.9 vs. 10.7 months), the combined PFS was inferior with everolimus followed by sunitinib versus sunitinib followed by everolimus ( 21.1 vs. 25.8 months). ${ }^{29}$ The median OS also favored sunitinib followed by everolimus, rather than the reverse (32 vs. 22.4 months). In a 2015 trial, investigators evaluated bevacizumab in a four-arm first-line study with bevacizumab monotherapy versus bevacizumab, temsirolimus versus bevacizumab, and sorafenib versus sorafenib and temsirolimus. There was no notable improvement in the primary endpoint of PFS, but toxicity was significantly greater in the combination arms. Forty-four percent of patients in the bevacizumab monotherapy arm had grade 3-5 AEs compared with 77\%-84\% of those in the combination arms. ${ }^{30}$ After encouraging phase II results, a phase III trial studied IMA901, a vaccine of 10 tumor-associated peptides, in combination with sunitinib in previously untreated mRCC. Although the peptide vaccine was well tolerated, there was no improvement in clinical outcomes compared with sunitinib monotherapy. ${ }^{31}$

\section{Preferred First-Line Agent}

HDIL-2, sorafenib, sunitinib, pazopanib, and bevacizumab with interferon are currently approved agents for previously untreated mRCC. Temsirolimus is approved for patients with $\mathrm{mRCC}$ with three or more poor prognosis criteria. To help elucidate the preferred agent, Motzer et $\mathrm{al}^{32}$ compared pazopanib to sunitinib in the first-line setting in the randomized COMPARZ trial. Although there was a greater ORR with pazopanib (31\% vs. $25 \%$ ), there was no difference in PFS (8.4 vs. 9.5 months) or OS (28.3 vs. 29.1 months). Although the 
rates of dose reduction and drug discontinuation because of AEs were similar in both arms, health-related quality-of-life scores were worse in the sunitinib arm and were mainly driven by fatigue, mouth and throat soreness, and hand-foot syndrome. ${ }^{32}$ The PISCES study, an innovative double-blind randomized controlled cross-over trial assessing treatment preference for pazopanib versus sunitinib, was undertaken with the primary endpoint being patient preference for a specific treatment as determined by a questionnaire developed by mRCC clinicians administered at the end of the treatment period. Utilizing 10-week treatment courses with a 2-week washout, the study showed improvement in patient preference (70\% vs. $22 \%)$ as well as clinician preference, a secondary endpoint, $(61 \%$ vs. $22 \%$ ) favoring pazopanib. ${ }^{33}$ Importantly, this is a relatively small study with a high attrition rate and has been criticized for administering the questionnaire immediately prior to the sunitinib rest period. ${ }^{34}$ Notably, there have not been any other trials comparing agents currently approved in the first-line therapy setting. Results from the CABOSUN trial may add another layer of complex decision making if they lead to the approval of cabozantinib for the first-line setting. Furthermore, the treatment landscape in the first-line therapy setting is expected to undergo major shifts with expected approval of multiple agents, based on results of numerous ongoing first-line clinical trials (Table 2).

As we await the data from these ongoing trials, we suggest HDIL-2 as the preferred agent for patients with excellent performance status, clear cell histology, and intact organ function (regardless of prognostic risk categorization), because HDIL-2 is currently the only agent shown to be associated with durable responses for approximately $10 \%$ of patients. We prefer sunitinib or pazopanib for patients in the good and intermediate risk categories who are not candidates for HDIL-2, based on equivalent survival outcomes in a randomized controlled trial comparing these agents. Although there is no randomized trial comparing the combination of bevacizumab and interferon to pazopanib or sunitinib, this remains a reasonable regimen to consider in front-line mRCC. For patients in the poor prognosis category, temsirolimus is approved but is associated with modest survival benefits and requires weekly intravenous administration, which may not be desirable or feasible for many patients. For these patients, sunitinib or pazopanib are the preferred options, based on the results of the COMPARZ trial in which clinical outcomes were similar with both agents. ${ }^{32}$

\section{SECOND-LINE THERAPY AND BEYOND}

Headlined by the 2005 FDA approval of sorafenib for patients previously treated with cytokine therapy, antiangiogenic agents have transformed treatment of mRCC salvage therapy over the past decade. After the TARGET study showed improvement in PFS over placebo (5.5 vs. 2.8 months; $\mathrm{p}<.001$ ), sorafenib became the first VEGF-TKI approved for mRCC. ${ }^{8}$ This was followed by the RECORD- 1 trial, which investigated everolimus versus placebo for patients who progressed after treatment with sunitinib or sorafenib. Patients in the everolimus-treated arm showed improved PFS (4.9 vs. 1.9 months; $\mathrm{p}<.001$ ), but everolimus was also associated with increased rates of stomatitis, rash, and fatigue. Similar to temsirolimus studies, there was also an increase in pneumonitis. ${ }^{15}$ In the aforementioned phase III VEG105192 study, pazopanib was also compared with placebo for patients in the first-line setting and for those who had progressed while receiving cytokine treatment and improved PFS in both subsets (9.2 vs. 4.2 months; $\mathrm{p}<.001) .{ }^{11}$ 
In 2012, the AXIS investigators published results from a study comparing axitinib to sorafenib for 723 patients with $\mathrm{mRCC}$ who had progressed with previous systemic therapy (35\% of whom were treated with cytokines; the rest received prior treatment with sunitinib, bevacizumab plus interferon, or temsirolimus). Initial results showed that the axitinib arm had improved PFS of 2 months compared with the sorafenib arm (6.7 vs. 4.7 months; onesided $\mathrm{p}<.0001$ ), which improved in the updated results (8.3 vs. 5.7 months; one-sided $\mathrm{p}<$. 0001). There was a notable improvement in PFS among subsets of patients previously treated with cytokines (12.2 vs. 8.2 months) or those treated previously with sunitinib (6.5 vs. 4.4 months), but not among those who had prior treatment with bevacizumab and interferon or temsirolimus. Overall, ORR was also improved in the axitinib arm (23\% vs. $12 \%)$, but OS was similar in both arms. AEs were also similar in both arms. ${ }^{17,18}$

Temsirolimus was also compared with sorafenib in a study of 512 patients who had previously progressed while taking sunitinib. There was no difference in PFS, but median OS favored the sorafenib arm (16.6 vs. 12.3 months). ${ }^{35}$

The next paradigm shift occurred in 2015, when three novel agents showed improved outcomes in the salvage setting. Nivolumab, a monoclonal antibody directed against the PD-1 receptor, is an immune checkpoint inhibitor that works to reverse tumor-induced immune suppression and stimulate antitumor immunity. Nivolumab was first approved for use in metastatic melanoma followed by non-small cell lung cancer, when a landmark Checkmate025 study compared nivolumab to everolimus in the second-line setting for 821 patients after progression on one or two previous antiangiogenic agents. Although PFS was similar in both arms (4.6 vs. 4.4 months), the primary endpoint OS was superior in the nivolumab arm (25.0 vs. 19.6 months; HR for death, $0.73 ; \mathrm{p}=.002)$. ORR was also greater in the nivolumab arm (25\% vs. $5 \%$ ) and there were significantly fewer grade 3-4 AEs (19\% vs. $37 \%) .{ }^{19}$ Another landmark study, the METEOR trial, investigated cabozantinib versus everolimus for 658 patients who had progressed after antiangiogenic therapy directed against VEGF. Sixty-nine percent of patients had only received one prior treatment, whereas the remaining patients had received at least two prior therapies. Cabozantinib resulted in improved in PFS (7.4 vs. 3.8 months; $p<.001)$, ORR (17\% vs. 3\%; p <.001), and OS (21.4 vs. 16.5 months; HR, $0.66 ; \mathrm{p}=.00026$ ). Remarkably, treatment with cabozantinib resulted in the longest PFS in the post-VEGF/TKI salvage monotherapy setting reported to date. More than $99 \%$ of patients in both arms reported an AE of any grade, but there was a greater incidence of grade 3-4 AEs in the cabozantinib arm (68\% vs. 58\%). More frequent grade 34 AEs with cabozantinib included hand-foot syndrome, hypertension, diarrhea, nausea, and thromboembolic events. ${ }^{20}$ Also reported in 2015, a phase II study investigated treatment with lenvatinib (a TKI of VEGFR1-VEGFR3, fibroblast growth factor receptors 1-4, platelet-derived growth factor receptor $a$, RET and KIT) for 153 patients with $\mathrm{mRCC}$ who had progressed after VEGF-targeted therapy. Patients were randomized into three arms: combination lenvatinib and everolimus versus lenvatinib monotherapy versus everolimus monotherapy. Respectively, PFS (14.6 vs. 7.4 vs. 5.5 months) and OS (25.5 vs. 18.4 vs. 15.4 months) were greater in the combination arm but only met statistical significance for the primary endpoint, which was PFS for patients who received lenvatinib plus everolimus versus everolimus monotherapy. Combination therapy was more toxic than everolimus monotherapy (grade 3-4 AEs, $71 \%$ vs. 50\%) with significantly greater diarrhea in the 
combination arm..$^{22}$ These three studies led to FDA approvals for nivolumab, cabozantinib, and lenvatinib in combination with everolimus for patients with mRCC for which an antiangiogenic agent failed previously. Notably, these agents are only approved after VEGFdirected treatment failure and are not approved for those patients with $\mathrm{mRCC}$ for which immune therapy failed.

\section{ONGOING CLINICAL TRIALS FOR UNTREATED MRCC}

Although the landscape of second-line and salvage therapy is rapidly evolving, there are many clinical trials at various stages investigating first-line therapy for mRCC (Table 2).

\section{Immunotherapy: Checkpoint Inhibitors}

Numerous immunotherapy agents are under active investigation in the first-line setting for mRCC, including seven trials investigating immune checkpoint inhibitors (Fig. 2). In a phase IIIB/IV trial (), nivolumab is being studied in combination with ipilimumab, a checkpoint inhibitor directed against CTLA-4. Nivolumab is also being studied in two phase II trials ( and ). The aforementioned trial plans to enroll 650 patients and is comparing nivolumab with ipilimumab versus nivolumab with BMS-986016, a monoclonal antibody checkpoint inhibitor directed against lymphocyte activation gene-3 (LAG-3). The latter trial is enrolling 244 patients and comparing nivolumab to sunitinib or pazopanib after treating patients for 10-12 weeks with a TKI. Pembrolizumab, another PD-1 monoclonal antibody, is currently being studied in combination with axitinib compared with sunitinib in a phase III trial of 840 patients and completion is expected in 2019 (). Another phase III trial of 735 patients () is comparing the combination of pembrolizumab and lenvatinib against combination lenvatinib and everolimus as well as sunitinib monotherapy. Investigators are also enrolling patients for first-line studies of checkpoint inhibitors targeting PD-L1 (avelumab and atezolizumab). In a phase III trial with 583 patients (), avelumab is being studied in combination with axitinib versus the comparator sunitinib.

\section{Selected Novel Agents in Development}

AGS-003 is a personalized immunotherapy of mature autologous dendritic cells that are coelectroporated with both synthetic RNA and the patient's tumor RNA. AGS-003 was designed to achieve the immunomodulatory effects of HDIL-2 with a more favorable toxicity profile, and AGS-003 was studied in combination with sunitinib in a phase II study of 22 patients with low- or intermediate-risk $\mathrm{mRCC}$. There were no CRs, but nine patients had a partial response and the median PFS and OS were encouraging (11.2 and 30.2 months, respectively). Notably, OS exceeded 5 years for five patients (24\%), with two patients (approximately 10\%) achieving durable responses for more than 5 years. ${ }^{36}$ The phase III ADAPT trial () has completed accrual and results are currently awaited. LY2510924 (X4P-001) is a novel cyclic peptide that inhibits CXCR-4, a chemokine receptor shown to be important in tumorgenesis. ${ }^{37}$ A phase II trial () comparing monotherapy with sunitinib versus sunitinib with LY2510924 is enrolling 108 patients, with completion anticipated in 2017. In a small phase I trial () with 18 patients, researchers are also investigating a polyvalent immune vaccine called HyperAcute-Renal, which is composed of an allogenic renal cell carcinoma (RCC) cell that has been genetically modified to express 1,3- 
galactosyltransferase with the intention to augment cellular immune responses. Hypoxiainducible factors (HIFs) are transcription factors activated in hypoxic states and by loss of von Hippel-Lindau function. HIF-1a and HIF-2 $a$ overexpression has long been known to be associated with malignant disease. ${ }^{38,39}$ In particular, HIF has been implicated in both tumorigenesis and angiogenesis in mRCC (Fig. 1). ${ }^{40}$ In another trial (), PT2385, a recently developed direct antagonist of HIF-2 $a$, is being investigated as a monotherapy and in combination with either cabozantinib or nivolumab for patients with mRCC who have progressed while taking previous therapy. A very similar agent, PT2399, has shown antitumor activity in a mouse model. ${ }^{40}$

Dalantercept, an activin receptor-like kinase 1 inhibitor that targets angiogenesis, is being tested in combination with axitinib for patients who progressed despite previous VEGF/TKI treatment. Phase II results of the DART study reported no dose-limiting toxicities among the 29 patients treated, with an ORR of $25 \%$ and PFS of 8.3 months. ${ }^{41}$ Part II of the study is a randomized trial () in which this combination is being compared with axitinib alone; the study is currently enrolling 174 patients, with completion estimated in 2018. Another trial () is combining a monoclonal antibody against endoglin, TRC105, with axitinib compared with axitinib monotherapy for 168 patients with mRCC. Endoglin is a glycoprotein expressed on endothelial cells and plays an important role in angiogenesis and tumorgenesis. ${ }^{42}$

\section{ROLE OF SURGERY IN THE METASTATIC SETTING}

\section{Cytoreductive Nephrectomy}

Most patients with mRCC who have been enrolled in systemic therapy clinical trials on $\mathrm{mRCC}$ have had cytoreductive nephrectomy $(\mathrm{CN})$ performed. In addition to palliation of pain, hematuria, and paraneoplastic syndromes, $\mathrm{CN}$, if clinically feasible, is likely to improve outcomes for carefully selected patients. $\mathrm{CN}$ has been a standard of care since two landmark trials showed an improvement in OS (17 vs. 7 months; HR, 0.54) for patients who underwent $\mathrm{CN}$ prior to therapy. ${ }^{43,44}$ Although both studies used interferon as the systemic therapy, an updated retrospective cohort of 15,390 patients treated with antiangiogenic targeted therapies reported similar survival data for those who underwent a CN (median OS of 17.1 vs. 7.7 months; $\mathrm{p}<.001) .{ }^{45}$ In a different retrospective cohort from the Surveillance, Epidemiology, and End Results database, investigators found that of 7,143 patients with reported $\mathrm{mRCC}$ treated with antiangiogenic targeted therapy, $37 \%$ underwent $\mathrm{CN}$. Those who underwent a CN had improved 1-year OS (61\% vs. 22\%; HR, 0.40), which was influenced by many factors, including a greater likelihood of having localized disease and younger age. ${ }^{46}$ In a retrospective IMDC cohort in the targeted therapy era, investigators performed a multivariate analysis of 314 patients, 201 of whom underwent $\mathrm{CN}$. Again, the IMDC investigators noted improvement in OS with CN (19.8 vs. 9.4 months; $p<.01)$, but this benefit was greatest for those with a favorable and intermediate prognosis. ${ }^{47}$ Given the inherent biases of these retrospective studies, a prospective study (CARMENA, ) is investigating $\mathrm{CN}$ by comparing sunitinib with or without $\mathrm{CN}$ in previously untreated patients with mRCC. CARMENA has a planned sample size of 700 participants with eligibility including biopsy proven $\mathrm{mRCC}$ and an ECOG $0-1$. The primary endpoint is OS. 
Although prospective results are pending, it is paramount that the treatment decision for $\mathrm{CN}$ be based on an individual patient's performance and prognostic status and made in conjunction with multidisciplinary input. Although no specific guidelines yet exist, a reasonable approach may be to exclude those who did not benefit from $\mathrm{CN}$ in the IMDC study. These included those with at least four poor IMDC prognostic factors: time from diagnosis to treatment less than 1 year, Karnofsky performance score less than 80, anemia, neutrophilia, and thrombocytosis. In addition, those with anticipated OS shorter than 1 year may not benefit from CN. ${ }^{47,48}$ It is also important to consider the probability of successfully debulking of adequate portion of the tumor.

\section{Metastasectomy}

Similar to CN, the role of metastasectomy for patients with $\mathrm{mRCC}$ is nuanced based on underlying patient characteristics. In a 1998 study of 278 patients with recurrent RCC, investigators found that $44 \%$ of patients were able to have long-term remission with oligometastasectomy (based on 5-year OS). Favorable features for response included a solitary metastatic organ site, the lung as the metastatic site, and age younger than 60 . Those with the brain as a solitary metastatic site had inferior outcomes to oligometastasectomy than those with lung (5-year OS, $18 \%$ vs. 54\%; p <.05). ${ }^{49}$ A 2011 study of 887 patients who underwent nephrectomy for RCC who later developed lung-confined metastatic lesions also showed impressive 5-year OS with complete versus incomplete resection (73.6\% vs. 19.0\%; $\mathrm{p}<.001) .{ }^{50}$ The same study also showed a survival advantage, although less robust, favoring complete over incomplete resection for patients with extrapulmonary metastatic lesions (5year OS, $32.5 \%$ vs. $12.4 \%$; $<$.001).$^{50}$ Other studies have shown improvement for resection of symptomatic bone lesions as well as complete resection of visceral metastases to the liver, thyroid, and pancreas. ${ }^{51-54}$ A randomized, double-blind placebo-controlled study, E2810 (), is currently enrolling patients to study whether pazopanib improves outcomes for individuals who successfully undergo complete resection of metastatic lesions.

\section{Adjuvant Therapy}

Thus far, adjuvant therapy results have been disappointing in RCC. A 2003 study of 69 patients with locally advanced or metastatic RCC treated with adjuvant HDIL-2 showed no benefit in disease-free survival (DFS) compared with a similar cohort of patients followed with observation. ${ }^{55}$ Recently, the Eastern Cooperative Oncology Group (ECOG) 2805 trial investigated sunitinib or sorafenib or placebo as adjuvant therapy for 1,943 patients with resected non-metastatic RCC. Unfortunately, results revealed that neither sorafenib or sunitinib improved DFS compared with placebo. ${ }^{56}$ In the S-TRAC adjuvant study of 615 patients with resected non-metastatic RCC, sunitinib significantly improved DFS compared with placebo ( 6.8 vs. 5.6 years; HR, 0.76 ); however, the duration of improvement in median DFS was similar to the duration of adjuvant therapy with sunitinib. ${ }^{57}$ Notably, per independent central review, the magnitude of benefit was higher with adjuvant sunitinib for patients with a higher risk of recurrence, defined as those with T3 tumors, without or with undetermined nodal involvement, with Fuhrman grade 2 or higher tumors, and with a ECOG performance score of 1 or higher; or those with a T4 tumor, local nodal involvement, or both. For these patients, DFS was significantly improved with sunitinib versus placebo (6.2 vs. 4.0 years; $\mathrm{HR}, 0.74 ; 95 \% \mathrm{CI}, 0.55-0.99 ; \mathrm{p}=.04)$. The OS results need to mature on 
further follow-up to see whether systemic anti-VEGF therapy actually effects a cure for any patient with mRCC. Recently reported preliminary results of the PROTECT study () with adjuvant pazopanib also failed to reveal a DFS benefit over placebo. A phase III trial through the National Clinical Trials Network, PROSPER RCC, was activated in 2017 and is comparing neoadjuvant and adjuvant nivolumab versus observation in localized RCC, with the hypothesis that prenephrectomy administration may improve T-cell response. In addition, phase III adjuvant therapy trials with atezolizumab and pembrolizumab are ongoing and continue to use placebo as the control arm.

\section{SEQUENCING PARADIGM}

To date, no randomized trials have yet established the optimal sequence of therapy in renal cancer. Most of the targeted therapies have been established in phase III trials that were conducted within the same timeframe, so very few were done with comparisons to another agent. The COMPARZ trial showed that pazopanib was noninferior to sunitinib, so either could be considered as a front-line therapy option. ${ }^{32}$ Randomized front-line trials comparing either axitinib or tivozanib to sorafenib did not reveal an advantage in efficacy. ${ }^{27,28}$ Of the previous trials, only COMPARZ used sunitinib for the comparator arm and others used sorafenib. Contemporary ongoing first-line studies of nivolumab and ipilimumab, atezolizumab and bevacizumab, pembrolizumab and axitinib, avelumab and axitinib, and lenvatinib and pembrolizumab are all using sunitinib as the control (Table 2). As discussed above, the CABOSUN trial also used sunitinib as the comparator against cabozantinib in the first-line setting. As the only therapy with decades of follow-up, HDIL-2 is associated with durable responses for $10 \%$ of patients and remains a treatment consideration; however, HDIL-2 requires carefully selecting patients with robust performance status and organ function, which limits its use.

Second-line therapies were also established in clinical trials that were conducted in parallel. Axitinib showed improved PFS compared with sorafenib, and everolimus showed better efficacy than placebo for patients previously treated with VEGF-directed therapies. All recent studies for the pretreated $\mathrm{mRCC}$ population have used everolimus as the comparator arm. Nivolumab, cabozantinib, and lenvatinib plus everolimus each revealed superior efficacy in response rates and OS. Hence, a contemporary therapy sequence pattern has emerged of sunitinib or pazopanib followed by nivolumab or cabozantinib or axitinib. Generally, if nivolumab is used in the second line, cabozantinib or axitinib is used in the third line or later and vice versa. Given higher toxicities but relatively higher response rates and PFS of the lenvatinib plus everolimus combination, this may be reserved for patients with rapidly progressive high-volume disease in the second-line therapy setting, or after disease progression following other monotherapies. It is postulated that the therapeutic sequence will undergo a dramatic change based on the results of first-line therapy trials (Table 2). Figure 3 provides an overview of current and potential future therapeutic sequencing in $\mathrm{mRCC}$.

A biomarker selection process would be a helpful tool; however, no predictive markers have been validated to date. Recent reports on BAP-1, SETD2, and PBRM-1 as potential prognostic and predictive biomarkers may foster the possibility of impacting risk profiling. ${ }^{58}$ 
Even PD-L1 expression, a useful marker in other malignancies, has failed to predict the efficacy of immune therapy in mRCC. Mutation load and the neutrophil-to-lymphocyte ratio are promising biomarkers to predict response to immune therapy within mRCC. ${ }^{59 ، 61}$ Novel targets involving resistance pathways such as CXCR-4 (X4P-001) and HIF-2 (PT2385) and immune modulators such as LAG-3 inhibitors (BMS-986016) are under clinical investigation and therapeutic development.

\section{CONCLUSION}

The field of kidney cancer has surprisingly thrived on targeted therapy, despite the disease being notorious for its heterogeneity. New advances have improved response rates, OS, and treatment-related toxicities for patients with mRCC. Although new discoveries in targeted therapies are paralleled by those in immunotherapy, the treatment paradigm continues to evolve. Despite the FDA's approval of multiple agents, clinical trials should continue to be the mainstay of treatment in mRCC, because every advance to date can be attributed to patient participation in therapeutic studies.

\section{References}

1. Fyfe G, Fisher RI, Rosenberg SA, et al. Results of treatment of 255 patients with metastatic renal cell carcinoma who received high-dose recombinant interleukin-2 therapy. J Clin Oncol. 1995;13:688-696. [PubMed: 7884429]

2. RI Fisher, Rosenberg SA, Fyfe G Long-term survival update for high-dose recombinant interleukin-2 in patients with renal cell carcinoma. Cancer J Sci Am. 2000;6 (Suppl 1):S55-S57. [PubMed: 10685660]

3. Yang JC, Sherry RM, Steinberg SM, et al. Randomized study of high-dose and low-dose interleukin-2 in patients with metastatic renal cancer. J Clin Oncol. 2003;21:3127-3132. [PubMed: 12915604]

4. Clark JI, Wong MK, Kaufman HL et al. Impact of sequencing targeted therapies with high-dose interleukin-2 immunotherapy: an analysis of outcome and survival of patients with metastatic renal cell carcinoma from an on-going observational IL-2 clinical trial: PROCLAIM(SM). Clin Genitourin Cancer. 2017; 15:31-41.e4. [PubMed: 27916626]

5. Stenehjem DD, Toole M, Merriman J, et al. Extension of overall survival beyond objective responses in patients with metastatic renal cell carcinoma treated with high-dose interleukin-2. Cancer Immunol Immunother. 2016;65:941-949. [PubMed: 27277816]

6. Medical Research Council Renal Cancer Collaborators. Interferon-a and survival in metastatic renal carcinoma: early results of a randomised controlled trial. Lancet. 1999;353:14-17. [PubMed: 10023944]

7. Gnarra JR, Tory K, Weng Y, et al. Mutations of the VHL tumour suppressor gene in renal carcinoma. Nat Genet. 1994;7:85-90. [PubMed: 7915601]

8. Escudier B, Eisen T, Stadler WM, et al.; TARGET Study Group. Sorafenib in advanced clear-cell renal-cell carcinoma. N Engl J Med. 2007;356:125-134. [PubMed: 17215530]

9. Motzer RJ, Hutson TE, Tomczak P, et al. Sunitinib versus interferon alfa in metastatic renal-cell carcinoma. N Engl J Med. 2007;356:115-124. [PubMed: 17215529]

10. Escudier B, Pluzanska A, Koralewski P, et al.; AVOREN Trial investigators. Bevacizumab plus interferon alfa-2a for treatment of metastatic renal cell carcinoma: a randomised, double-blind phase III trial. Lancet. 2007;370:2103-2111. [PubMed: 18156031]

11. Sternberg CN, Davis ID, Mardiak J, et al. Pazopanib in locally advanced or metastatic renal cell carcinoma: results of a randomized phase III trial. J Clin Oncol. 2010;28:1061-1068. [PubMed: 20100962] 
12. Hudes G, Carducci M, Tomczak P, et al.; Global ARCCTrial. Temsirolimus, interferon alfa, or both for advanced renal-cell carcinoma. N Engl J Med. 2007;356:2271-2281. [PubMed: 17538086]

13. Motzer RJ, Hutson TE, Tomczak P, et al. Overall survival and updated results for sunitinib compared with interferon alfa in patients with metastatic renal cell carcinoma. J Clin Oncol. 2009;27:3584-3590. [PubMed: 19487381]

14. Escudier B, Bellmunt J, Negrier S, et al. Phase III trial of bevacizumab plus interferon alfa-2a in patients with metastatic renal cell carcinoma (AVOREN): final analysis of overall survival. J Clin Oncol. 2010;28:2144-2150. [PubMed: 20368553]

15. Motzer RJ, Escudier B, Oudard S, et al.; RECORD-1 Study Group. Efficacy of everolimus in advanced renal cell carcinoma: a double-blind, randomised, placebo-controlled phase III trial. Lancet. 2008;372:449-456. [PubMed: 18653228]

16. Motzer RJ, Escudier B, Oudard S, et al.; RECORD-1 Study Group. Phase 3 trial of everolimus for metastatic renal cell carcinoma: final results and analysis of prognostic factors. Cancer. 2010;116:4256-4265. [PubMed: 20549832]

17. Bl Rini, B Escudier, P Tomczak, et al. Comparative effectiveness of axitinib versus sorafenib in advanced renal cell carcinoma (AXIS): a randomised phase 3 trial. Lancet. 2011;378:1931-1939. [PubMed: 22056247]

18. Motzer RJ, Escudier B, Tomczak P, et al. Axitinib versus sorafenib as second-line treatment for advanced renal cell carcinoma: overall survival analysis and updated results from a randomised phase 3 trial. Lancet Oncol. 2013;14:552-562. [PubMed: 23598172]

19. Motzer RJ, Escudier B, McDermott DF, et al.; CheckMate 025 Investigators. Nivolumab versus everolimus in advanced renal-cell carcinoma. N Engl J Med. 2015;373:1803-1813. [PubMed: 26406148]

20. Choueiri TK, Escudier B, Powles T, et al.; METEOR Investigators. Cabozantinib versus everolimus in advanced renal-cell carcinoma. N Engl J Med. 2015;373:1814-1823. [PubMed: 26406150]

21. Choueiri TK, Escudier B, Powles J, et al.; METEOR investigators. Cabozantinib versus everolimus in advanced renal cell carcinoma (METEOR): final results from a randomised, open-label, phase 3 trial. Lancet Oncol. 2016;17:917-927. [PubMed: 27279544]

22. Motzer RJ, Hutson TE, Glen H, et al. Lenvatinib, everolimus, and the combination in patients with metastatic renal cell carcinoma: a randomised, phase 2, open-label, multicentre trial. Lancet Oncol. 2015;16:1473-1482. [PubMed: 26482279]

23. Hutson T, Dutcus C, Ren M, et al. Subgroup analyses and updated overall survival from the phase 2 trial of lenvatinib (LEN), everolimus (EVE), and LEN plus EVE in metastatic renal cell carcinoma (mRCC) In Hallek M (ed). Oncology Research and Treatment. Basel, Switzerland: Karger; 2016;318-319.

24. Choueiri TK, Halabi S, Sanford BL, et al. Cabozantinib versus sunitinib as initial targeted therapy for patients with metastatic renal cell carcinoma of poor or intermediate risk: the Alliance A031203 CABOSUN trial. J Clin Oncol. 2017;35:591-597. [PubMed: 28199818]

25. Hennessy BT, Smith DL, Ram PT, et al. Exploiting the PI3K/AKT pathway for cancer drug discovery. Nat Rev Drug Discov. 2005;4:988-1004. [PubMed: 16341064]

26. Escudier B, Szczylik C, Hutson TE, et al. Randomized phase II trial of first-line treatment with sorafenib versus interferon Alfa-2a in patients with metastatic renal cell carcinoma. J Clin Oncol. 2009;27:1280-1289. [PubMed: 19171708]

27. Hutson TE, Lesovoy V, Al-Shukri S, et al. Axitinib versus sorafenib as first-line therapy in patients with metastatic renal-cell carcinoma: a randomised open-label phase 3 trial. Lancet Oncol. 2013;14:1287-1294. [PubMed: 24206640]

28. Motzer RJ, Nosov D, Eisen T, et al. Tivozanib versus sorafenib as initial targeted therapy for patients with metastatic renal cell carcinoma: results from a phase III trial. J Clin Oncol. 2013;31:3791-3799. [PubMed: 24019545]

29. Motzer RJ, Barrios CH, Kim TM, et al. Phase II randomized trial comparing sequential first-line everolimus and second-line sunitinib versus first-line sunitinib and second-line everolimus in patients with metastatic renal cell carcinoma. J Clin Oncol. 2014;32:2765-2772. [PubMed: 25049330] 
30. Flaherty KT, Manola JB, Pins M, et al. BEST: a randomized phase II study of vascular endothelial growth factor, RAF kinase, and mammalian target of rapamycin combination targeted therapy with bevacizumab, sorafenib, and temsirolimus in advanced renal cell carcinoma-A trial of the ECOG-ACRIN Cancer Research Group (E2804). J Clin Oncol. 2015;33:2384-2391. [PubMed: 26077237]

31. Rini B, Stenzl A, Zdrojowy R, et al. 17LBA results from an open-label, randomized, controlled phase 3 study investigating IMA901 multipeptide cancer vaccine in patients receiving sunitinib as first-line therapy for advanced/metastatic RCC. Eur J Cancer. 2015;51 (Suppl 3):S718.

32. Motzer RJ, Hutson TE, Celia D, et al. Pazopanib versus sunitinib in metastatic renal-cell carcinoma. N Engl J Med. 2013;369:722-731. [PubMed: 23964934]

33. Escudier B, Porta C, Bono P, et al. Randomized, controlled, doubleblind, cross-over trial assessing treatment preference for pazopanib versus sunitinib in patients with metastatic renal cell carcinoma: PISCES study. J Clin Oncol. 2014;32:1412-1418. [PubMed: 24687826]

34. Pal SK, Vogelzang NJ. A "game of thrones" in metastatic renal cell carcinoma: vascular endothelial growth factor-tyrosine kinase inhibitors and mammalian target of rapamycin inhibitors battling for position. Clin Genitourin Cancer. 2013;11:1-4. [PubMed: 23276588]

35. Hutson TE, Escudier B, Esteban E, et al. Randomized phase III trial of temsirolimus versus sorafenib as second-line therapy after sunitinib in patients with metastatic renal cell carcinoma. $\mathrm{J}$ Clin Oncol. 2014;32:760-767. [PubMed: 24297950]

36. Amin A, Dudek AZ, Logan TF, et al. Survival with AGS-003, an autologous dendritic cell-based immunotherapy, in combination with sunitinib in unfavorable risk patients with advanced renal cell carcinoma (RCC): phase 2 study results. J Immunother Cancer. 2015;3:14. [PubMed: 25901286]

37. Peng SB, Zhang X, Paul D, et al. Identification of LY2510924, a novel cyclic peptide CXCR4 antagonist that exhibits antitumor activities in solid tumor and breast cancer metastatic models. Mol Cancer Ther. 2015;14:480-490. [PubMed: 25504752]

38. Zhong H, De Marzo AM, Laughner E, et al. Overexpression of hypoxia-inducible factor la in common human cancers and their metastases. Cancer Res. 1999;59:5830-5835. [PubMed: 10582706]

39. Talks KL, Turley H, Gatter KC, et al. The expression and distribution of the hypoxia-inducible factors HIF-Ia and HIF-2a in normal human tissues, cancers, and tumor-associated macrophages. Am J Pathol. 2000;157:411-421. [PubMed: 10934146]

40. Chen W, Hill H, Christie A, et al. Targeting renal cell carcinoma with a HIF-2 antagonist. Nature. 2016;539:112-117. [PubMed: 27595394]

41. Voss MH, Bhatt RS, Plimack ER, et al. The DART study: results from the dose-escalation and expansion cohorts evaluating the combination of dalantercept plus axitinib in advanced renal cell carcinoma. Clin Cancer Res. Epub 2016 Dec 28.

42. Dubinski W, Gabril M, Iakovlev VV, et al. Assessment of the prognostic significance of endoglin (CD105) in clear cell renal cell carcinoma using automated image analysis. Hum Pathol. 2012;43:1037-1043. [PubMed: 22204709]

43. Flanigan RC, Salmon SE, Blumenstein BA, et al. Nephrectomy followed by interferon alfa-2b compared with interferon alfa-2b alone for metastatic renal-cell cancer. N Engl J Med. 2001;345:1655-1659. [PubMed: 11759643]

44. Mickisch GH, Garin A, van Poppel H, et al.; European Organisation for Research and Treatment of Cancer (EORTC) Genitourinary Group. Radical nephrectomy plus interferon-alfa-based immunotherapy compared with interferon aIfa alone in metastatic renal-cell carcinoma: a randomised trial. Lancet. 2001;358:966-970. [PubMed: 11583750]

45. Hanna N, Sun M, Meyer CP, et al. Survival analyses of patients with metastatic renal cancer treated with targeted therapy with or without cytoreductive nephrectomy: a National Cancer Data Base study. J Clin Oncol. 2016;34:3267-3275. [PubMed: 27325852]

46. Abern MR, Scosyrev E, Tsivian M, et al. Survival of patients undergoing cytoreductive surgery for metastatic renal cell carcinoma in the targeted-therapy era. Anticancer Res. 2014;34:2405-2411. [PubMed: 24778051] 
47. Choueiri TK, Xie W, Kollmannsberger C, et al. The impact of cytoreductive nephrectomy on survival of patients with metastatic renal cell carcinoma receiving vascular endothelial growth factor targeted therapy. J Urol. 2011;185:60-66. [PubMed: 21074201]

48. Heng DY, Wells JC, Rini BI, et al. Cytoreductive nephrectomy in patients with synchronous metastases from renal cell carcinoma: results from the International Metastatic Renal Cell Carcinoma Database Consortium. Eur Urol. 2014;66:704-710. [PubMed: 24931622]

49. Kavolius JP, Mastorakos DP, Pavlovich C, et al. Resection of metastatic renal cell carcinoma. J Clin Oncol. 1998;16:2261-2266. [PubMed: 9626229]

50. Alt AL, Boorjian SA, Lohse CM, et al. Survival after complete surgical resection of multiple metastases from renal cell carcinoma. Cancer. 2011;117:2873-2882. [PubMed: 21692048]

51. Lin PP, Mirza AN, Lewis VO, et al. Patient survival after surgery for osseous metastases from renal cell carcinoma. J Bone Joint Surg Am. 2007;89:1794-1801. [PubMed: 17671020]

52. Alves A, Adam R, Majno P, et al. Hepatic resection for metastatic renal tumors: is it worthwhile? Ann Surg Oncol. 2003;10:705-710. [PubMed: 12839857]

53. Hegerova L, Griebeler ML, Reynolds JP, et al. Metastasis to the thyroid gland: report of a large series from the Mayo Clinic. Am J Clin Oncol. 2015;38:338-342. [PubMed: 23799287]

54. Tanis PJ, van der Gaag NA, Busch OR, et al. Systematic review of pancreatic surgery for metastatic renal cell carcinoma. Br J Surg. 2009;96:579-592. [PubMed: 19434703]

55. JI Clark, Atkins MB Urba WJ, et al. Adjuvant high-dose bolus interleukin-2 for patients with highrisk renal cell carcinoma: a cytokine working group randomized trial. J Clin Oncol. 2003;21:31333140. [PubMed: 12810695]

56. Haas NB, Manola J, Uzzo RG, et al. Adjuvant sunitinib or sorafenib for high-risk, non-metastatic renal-cell carcinoma (ECOG-ACRIN E2805): a double-blind, placebo-controlled, randomised, phase 3 trial. Lancet. 2016;387:2008-2016. [PubMed: 26969090]

57. Ravaud A, Motzer RJ, Pandha HS, et al.; S-TRAC Investigators. Adjuvant sunitinib in high-risk renal-cell carcinoma after nephrectomy. N Engl J Med. 2016;375:2246-2254. [PubMed: 27718781]

58. Pawtowski R, Mühl SM, Sulser T, et al. Loss of PBRM1 expression is associated with renal cell carcinoma progression. Int J Cancer. 2013;132:E11-E17. [PubMed: 22949125]

59. Nakamura Y, Kitano S, Takahashi A, et al. Nivolumab for advanced melanoma: pretreatment prognostic factors and early outcome markers during therapy. Oncotarget. 2016;7:77404-77415. [PubMed: 27764805]

60. Kuzman JA, Stenehjem DD, Merriman J, et al. Neutrophil-lymphocyte ratio as a predictive biomarker for response to high dose interleukin-2 in patients with renal cell carcinoma. BMC Urol. 2017;17:1. [PubMed: 28056941]

61. Ma W, Gilligan BM, Yuan J, et al. Current status and perspectives in translational biomarker research for PD-1/PD-L1 immune checkpoint blockade therapy. J Hematol Oncol. 2016;9:47. [PubMed: 27234522] 


\section{KEY POINTS}

- New discoveries and advances with antiangiogenic agents have changed the treatment paradigm for clear cell advanced and metastatic renal cell carcinoma.

- $\quad$ Based on improved outcomes, axitinib, nivolumab, cabozantinib, and combination lenvatinib plus everolimus are considered standard of care in the post-VEGF/TKI salvage therapy setting, with no clear consensus on how to optimally sequence these agents in the absence of head-to-head comparisons of these agents. Until validated molecular biomarkers predictive for the efficacy of specific agents are discovered, clinical decision making would be key to optimizing sequencing of these agents.

- $\quad$ Novel targeted therapies, immune checkpoint inhibitors, and immunemodulating drugs are currently under investigation, many in combination with VEGF pathway inhibitors, in the first-line setting and may replace sunitinib, pazopanib, and HDIL-2 as preferred first-line agents in the near future.

- Multidisciplinary input is important when considering the role of cytoreductive nephrectomies and metastatectomies in selected cases depending on overall prognosis and organ of involvement with metastatic disease.

- $\quad$ No efforts should be spared in providing access to clinical trials, facilitating approval of novel agents and combinations, and expediting validation of therapeutic biomarkers as well as exploring the optimal sequencing of the therapies. 


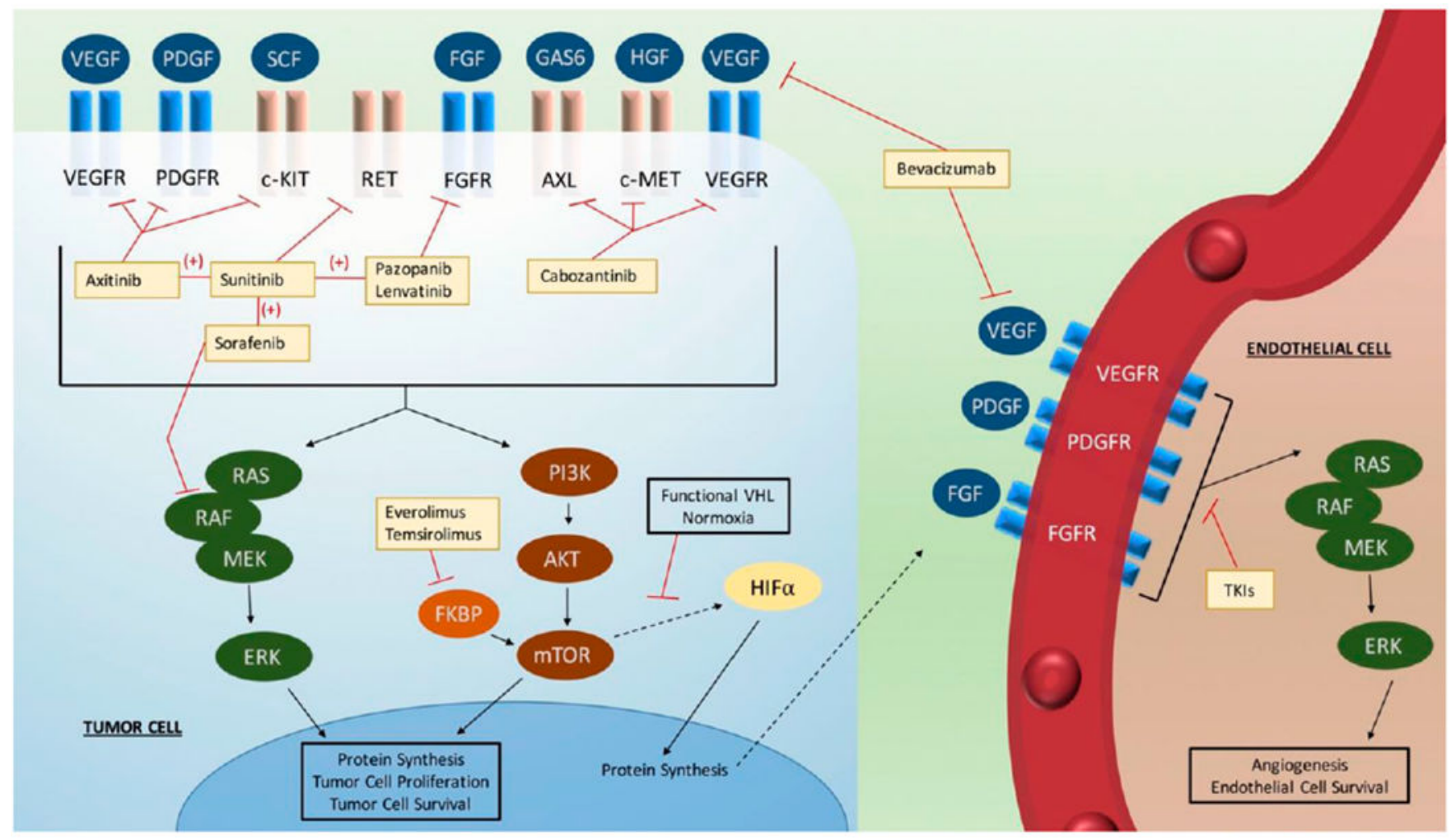

FIGURE 1. Mechanisms of Action of Targeted Therapies in Metastatic Renal Cell Carcinoma Ligand binding of RTKs leads to many downstream effects. Proangiogenic RTKs (VEGFR, PDGFR, and FGFR) are labeled in blue, whereas growth factor ligand binding to RTKs is colored orange. Simplified mechanisms of the MAPK pathway (left) and mTOR pathway (right) are labeled in the tumor cell. Activation of HIF-a occurs in states of hypoxia and through lack of inhibition from a nonfunctional VHL gene. It leads to synthesis of VEGF, PDGF, and FGF. This can lead to MAPK activation in endothelial cells, depicted on the left side of the diagram. Through FKBP is the site of action of mTOR inhibitors everolimus and temsirolimus and is labeled in orange. Bevacizumab is a monoclonal antibody directed against VEGF. TKIs include sorafenib, sunitinib, axitinib, pazopanib, cabozantinib, and Ienvatinib. The aforementioned TKIs inhibit multiple RTKs. RTKs of importance are inhibited by the following TKIs: axitinib (VEGFR, PDGFR, c-KIT), sunitinib (VEGFR, PDGFR, RET, c-KIT), sorafenib (VEGFR, PDGFR, RET, c-KIT, Raf kinases), pazopanib (VEGFR, PDGFR, FGFR, c-KIT, RET), Ienvatinib (VEGFR, PDGFR, FGFR, c-KIT, RET), and cabozantinib (VEGFR, c-MET, AXL, c-KIT, RET).

Abbreviations: ERK, extracellular signal-regulated kinase; FGF, fibroblast growth factor; FGFR, fibroblast growth factor receptor; FKBP, FK506-binding protein; GAS6, growth arrest-specific 6; HGF, hepatocyte growth factor; MAPK, mitogen-activated protein kinase; MEK, mitogen-activated protein kinase kinase; PDGF, platelet-derived growth factor; PDGFR, platelet-derived growth factor receptor; PI3K, phosphatidylinositol-3 kinase; RTK, receptor tyrosine kinase; $\mathrm{SCF}$, stem cell factor; TKI, tyrosine kinase inhibitor; VEGFR, VEGF receptor; VHL, von Hippel-Lindau. 


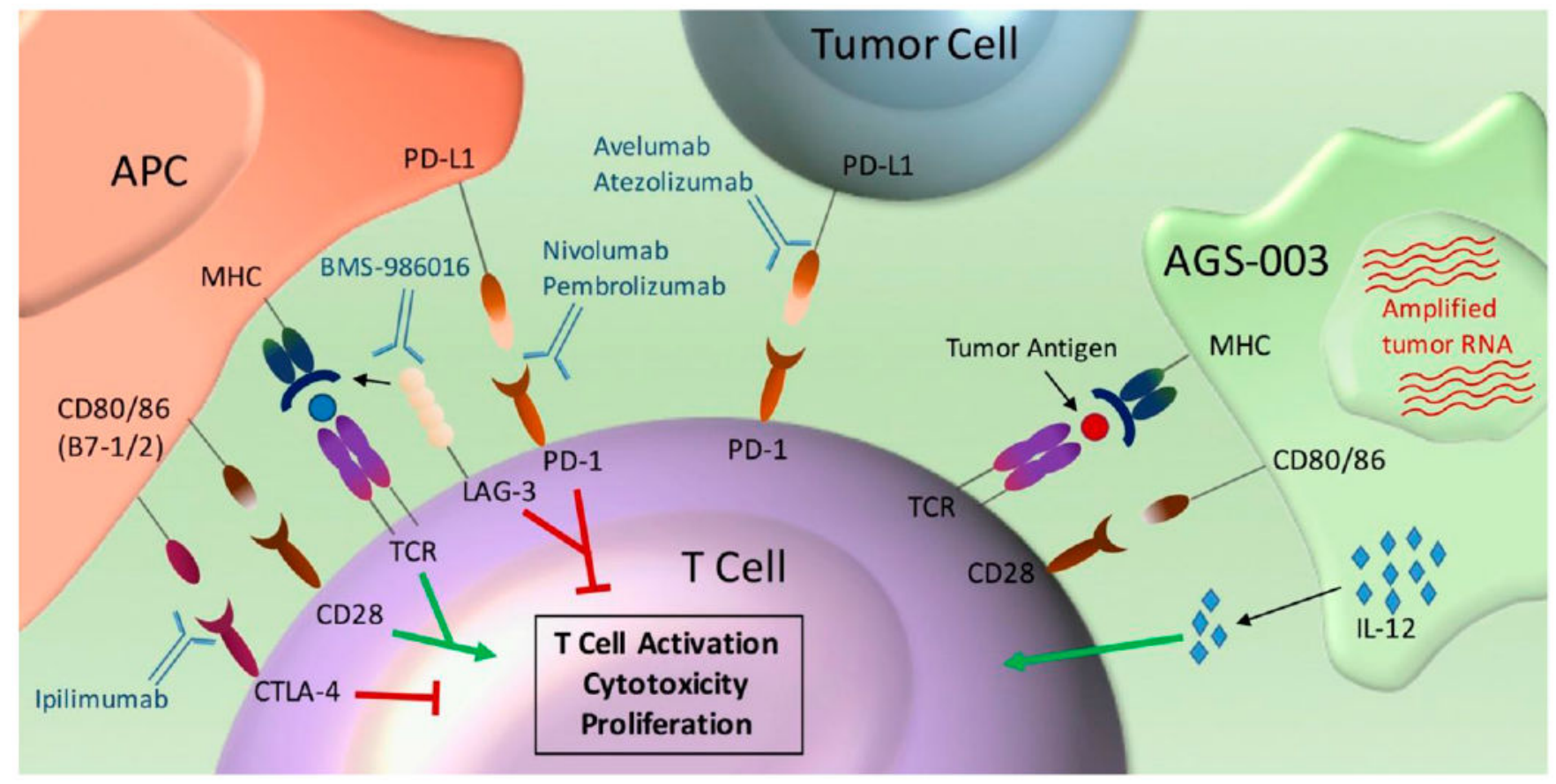

FIGURE 2. Mechanisms of Action of Immunotherapies for Metastatic Renal Cell Carcinoma Immune checkpoint inhibitors and AGS-003 are featured. Ipilimumab is a monoclonal antibody directed against CTLA-4 on T cells. Nivolumab and pembrolizumab inhibit PD-1 on both tumor and T cells, whereas avelumab and atezolizumab inhibit its ligand, PD-L1. BMS-986016 is a novel immune agent that targets LAG-3, preventing immune inhibition by preventing LAG-3 binding to its ligand, MHC class II receptor. AGS-003 is a mature dendritic cell that has been coelectroporated with tumor DNA and human CD40 ligand. By processing and then presenting tumor antigen, it binds both the TCR and CD28. A third signal leads to with IL-12 promotes memory T-cell development.

Abbreviations: IL, interleukin; MHC, major histocompatibility complex; TCR, T-cell receptor. 


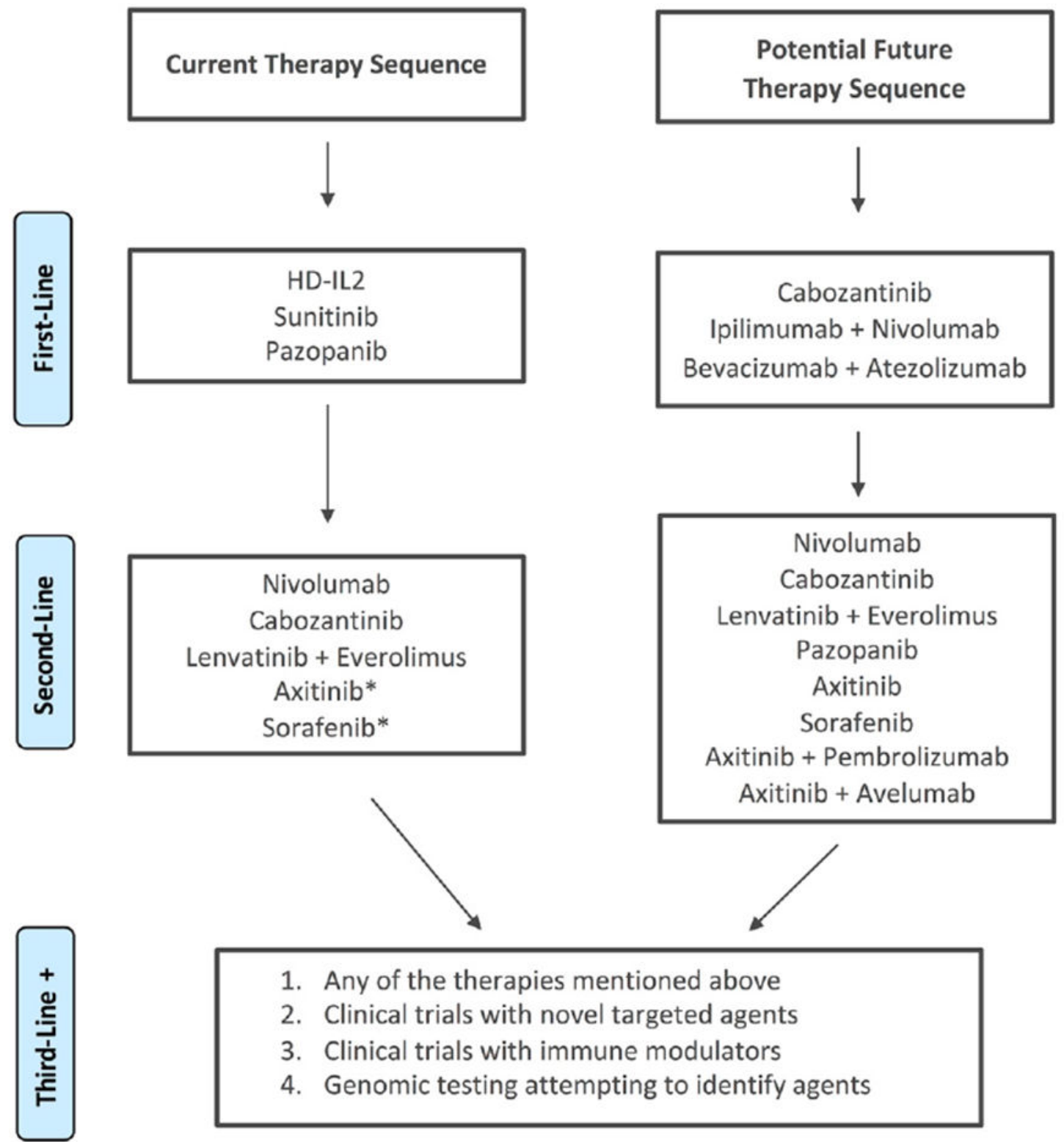

*Currently nivolumab, cabozantinib, and lenvatinib + everolimus are only approved after previous anti-angiogenic therapy. After failure of HDIL-2, patients should receive axitinib or sorafenib.

FIGURE 3. Sequencing Paradigm of Metastatic Renal Cell Carcinoma Asterisks indicate that nivolumab, cabozantinib, and lenvatinib plus everolimus are currently approved for use only after previous antiangiogenic therapy. After failure of HDIL-2, patients could receive axitinib or sorafenib.

Abbreviations: HDIL-2, high-dose interleukin-2. 


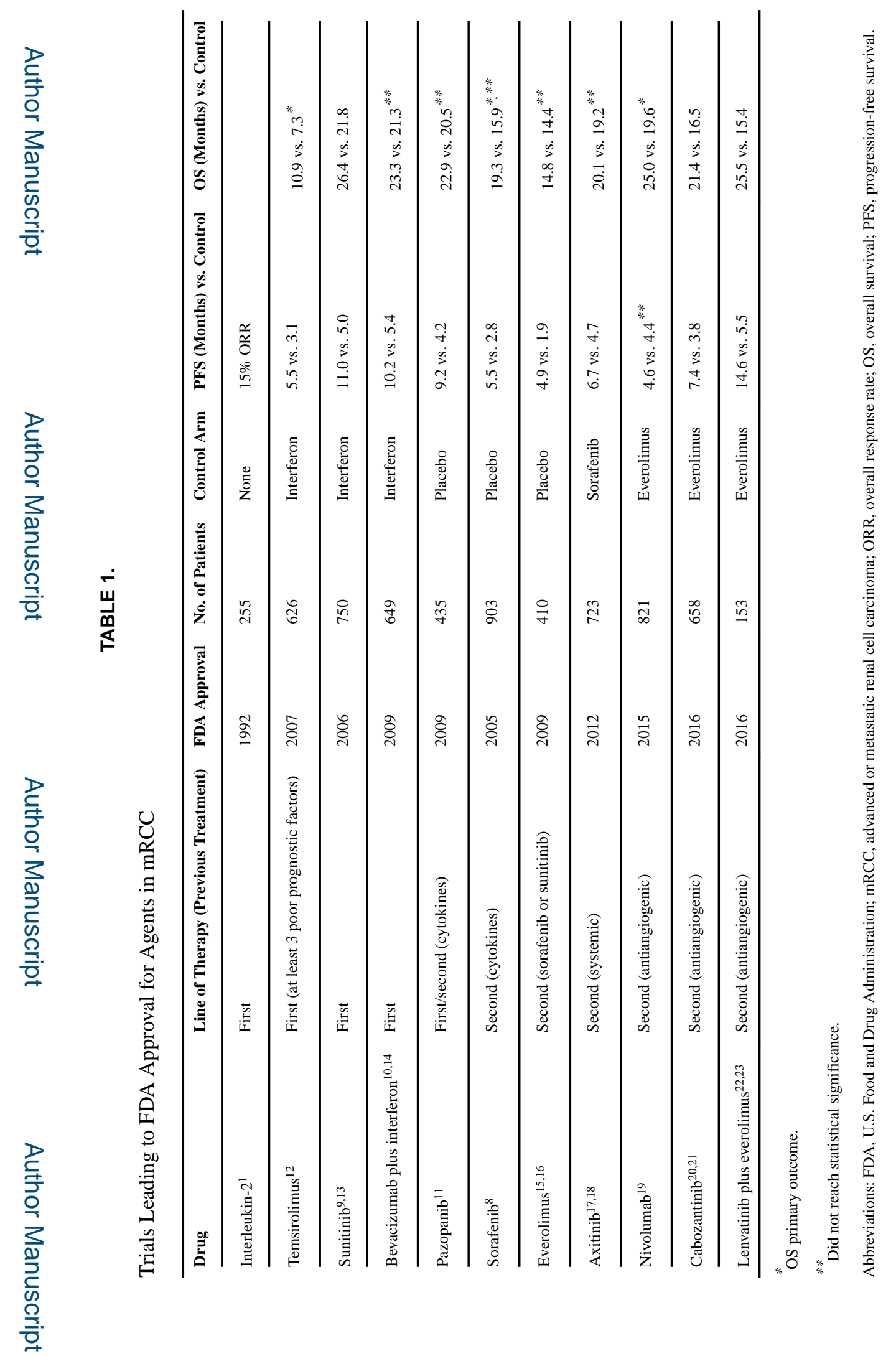




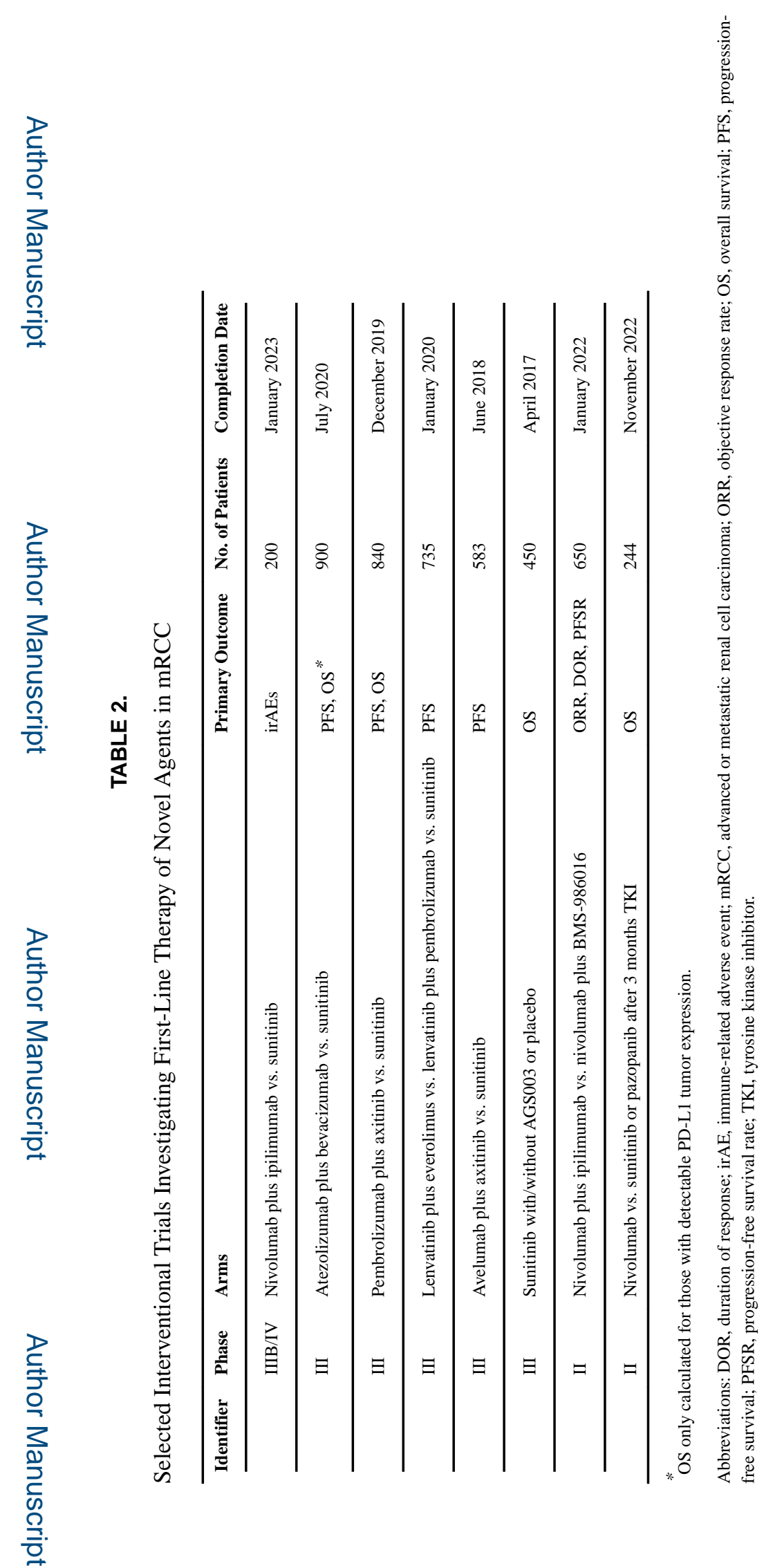

